\title{
Les usages de la biologie en sciences sociales
}

Comparaison entre le naturalisme socio-anthropologique du dixneuvième siècle et celui d'aujourd'hui

Dominique Guillo

\section{(2) OpenEdition}

12 Journals

Édition électronique

URL : http://journals.openedition.org/ress/1194

DOI : 10.4000/ress. 1194

ISSN : 1663-4446

Éditeur

Librairie Droz

\section{Édition imprimée}

Date de publication : 15 juin 2012

Pagination : 191-226

ISBN : 978-2-600-01613-1

ISSN : 0048-8046

\section{Référence électronique}

Dominique Guillo, «Les usages de la biologie en sciences sociales », Revue européenne des sciences sociales [En ligne], 50-1 | 2012, mis en ligne le 15 juin 2015, consulté le 10 décembre 2020. URL : http://journals.openedition.org/ress/1194; DOI : https://doi.org/10.4000/ress.1194 


\title{
LES USAGES DE LA BIOLOGIE EN SCIENCES SOCIALES
}

\section{COMPARAISON ENTRE LE NATURALISME SOCIO-ANTHROPOLOGIQUE DU DIX-NEUVIÈME SIĖCLE ET CELUI D'AUJOURD'HUI}

DOMINIQUE GUILLO

Centre Jacques Berque/CNRS

dominiqueguillo@yahoo.fr

\begin{abstract}
Résumé. Les théories socio-anthropologiques qui s'inspirent aujourd'hui des sciences de la vie sont volontiers regardées en sciences sociales comme des discours qui présentent de profondes affinités avec leurs homologues du dix-neuvième siècle. Dans de nombreuses études consacrées à ces doctrines, la référence à la biologie est considérée comme une trame discursive qui conduit nécessairement ceux qui la mobilisent à déployer des schèmes théoriques et idéologiques globalement semblables. Pourtant, lorsque l'on s'attache aux concepts biologiques mobilisés par ces théories, on se rend compte qu'ils sont fort différents, à un siècle de distance, la biologie ayant profondément modifié le regard qu'elle porte sur le vivant et sa diversité. Les sciences de la vie du dix-neuvième siècle condamnaient ceux qui s'en inspiraient pour expliquer l'homme, ses sociétés et ses cultures, à développer des discours historicistes et discriminatoires. Aujourd'hui, le naturalisme peut échapper à de tels discours, compte tenu du modèle biologique sur lequel il s'appuie.
\end{abstract}

Mots-clés: darwinisme, sciences de la vie, socio-anthropologie, naturalisme, biologie, déterminisme.

\begin{abstract}
The modern social anthropological theories which bear connections with the life sciences are still considered by social scientists as having deep affinities with their 19th century counterparts. Most studies of these works analyze the reference they make to biology as a discursive pattern leading those who resort to it to develop generally similar theoretical and ideological frameworks. Yet, when one focuses on the biological concepts they display, it appears that they are quite different from those circulating a century before, as biology itself has profoundly changed its perspective on life and diversity. Although borrowing from the $19^{\text {th }}$ century life sciences to explain Man, society and culture was once condemning one to develop historicist and discriminatory discourses, it is no longer the case given the biological model on which naturalism is based today.
\end{abstract}

Keywords: darwinism, life sciences, socio-anthropology, naturalism, biology, determinism. 
Les discours qui mobilisent la biologie pour expliquer les faits sociaux et culturels sont aujourd'hui vivement critiqués par les sciences sociales, dans le sillage de la polémique suscitée par la sociobiologie à partir du milieu des années 1970 (Wilson, 1975). Dans le concert de ces critiques, un mode d’argumentation est largement privilégié depuis une trentaine d’années. Il consiste à porter le regard sur le dix-neuvième siècle, période durant laquelle se réalisent les premiers mariages entre les sciences de la vie, la sociologie et l'anthropologie. L'objectif généralement affiché dans ces travaux critiques est de mettre au jour, à travers ce retour sur le passé, la persistance de schèmes idéologiques stables et identiques d'un siècle à l'autre - souvent, l'on évoque l'ultralibéralisme, le racisme, le sexisme, la justification des discriminations sociales - derrière un maquillage lexical d’allure scientifique. Les versions les plus ouvertement critiques reprennent ou retrouvent une thèse développée pour la première fois par Karl Marx et Friedrich Engels au sujet du darwinisme et de ses avatars socio-anthropologiques. Les sociologies et anthropologies d'inspiration biologisante d'hier et d'aujourd'hui s'appuieraient sur une conception idéologique de la nature dans laquelle les phénomènes biologiques sont peints, dans un premier temps, aux couleurs du capitalisme libéral; ce qui permettrait, en retour, de développer une rhétorique d’apparence scientifique, dans laquelle les inégalités sécrétées par le capitalisme peuvent apparaître comme fondées en nature (Sahlins, 1980; Berthoud [ed.], 1985; Tort, 1992).

L’objectif du présent article n'est pas de rejeter en bloc ces lectures critiques du naturalisme socio-anthropologique. Fort variées dans leurs attendus, leurs conclusions, comme dans leur méthodes, elles ont assurément permis d'éclairer certains aspects des conditions d'émergence et de diffusion de ces discours, et de montrer les connexions qui rattachent une partie d'entre eux à de telles idéologies. Le but que l'on se donne ici est de montrer les bénéfices que l'on peut attendre d'une démarche qui suit un cheminement inverse: non plus examiner comment un même fonds idéologique se perpétue dans les discours biologisants sur l'homme, la société et la culture au dix-neuvième siècle et 
aujourd'hui; mais comparer les propositions et les concepts biologiques généraux à travers lesquels sont formulés ces discours. En somme, ne plus centrer d'emblée l'analyse sur les partis pris normatifs, explicites ou implicites, et les liens qu'ils peuvent tisser entre les théories, mais sur les propositions positives formulées dans ces théories sur le vivant et la société ${ }^{2}$.

Sous un tel éclairage, des similitudes nettes, quoique rarement soulignées, apparaissent entre le naturalisme du dix-neuvième siècle et celui d'aujourd'hui. Tout d'abord, les diverses théories d'inspiration biologique sur l'homme et la société proposées durant chacune de ces périodes ne se réduisent pas à ce que l'on nomme ordinairement le naturalisme réductionniste ou le déterminisme biologique. Un naturalisme analogique, opposé au réductionnisme, s’y développe également. En réalité, les différents usages de la biologie peuvent être considérés, à chaque époque, comme autant de positionnements dans une cosmologie unitaire, qui en constitue le sous-bassement paradigmatique général.

Comme on va tenter de le montrer, la cosmologie naturaliste qui est propre au dix-neuvième siècle et celle qui est propre à la période contemporaine ont quelques points communs. Elles ont toutefois une différence sur laquelle il convient d'insister avec force, dans le cadre des débats actuels entre sciences sociales et sciences de la vie: les modèles biologiques à partir desquels elles sont respectivement bâties sont radicalement différents, bien que le nom de Darwin soit invoqué dans les unes comme dans les autres. Or ceci entraîne une conséquence épistémologique capitale: le naturalisme du dix-neuvième était condamné, par essence, à déployer une conception historiciste, hiérarchique et discriminatoire de l'homme; le naturalisme d’aujourd'hui peut y échapper, même si ce n'est pas toujours le cas.

2 La distinction effectuée ici entre les aspects positifs et normatifs des discours est purement méthodologique. II ne s'agit pas de considérer que les propositions constitutives d'un discours peuvent être séparées en deux groupes, les premières étant scientifiques, les secondes idéologiques. II s'agit simplement de considérer qu'il y a là deux registres argumentatifs différents - une proposition donnée pouvant se situer dans les deux registres, suivant le contexte de réception et l'angle rhétorique sous lequel elle est considérée - et que l'on peut, à titre de méthode analytique, choisir de suivre prioritairement l'un de ces registres, plutôt que l'autre, pour étudier un discours. 


\section{LES USAGES DE LA BIOLOGIE EN SCIENCES SOCIALES AU DIX-NEUVIÈME SIĖCLE La logique de l'organisation}

Les concepts biologiques mobilisés par les sociologues et les anthropologues du dix-neuvième siècle sont empruntés pour l'essentiel à un paradigme qui se déploie dans la discipline qui domine alors les sciences de la vie : l'anatomie comparée (Balan, 1979; Gayon, 1992 ; Guillo, 2003). Cet emprunt massif ne doit guère étonner. L’attrait exercé par ce paradigme dans le monde lettré de ce temps va en effet bien au-delà de la biologie, comme l'atteste, entre autres, l'immense retentissement de la «querelle des analogues»3, qui oppose, en I830, Georges Cuvier à Étienne Geoffroy Saint-Hilaire. Les sciences sociales naissantes - du moins celles qui prêtent attention à la biologie - empruntent à cette anatomie comparée trois concepts principaux, qu'elles portent à un haut degré d’abstraction : la classification, le développement et, surtout, notion centrale, l'organisation, à partir de laquelle est bâtie, comme on va le voir, la cosmologie intégratrice propre au naturalisme du dix-neuvième siècle.

\section{I. L'ANATOMIE COMPARÉE}

Une science des principes et des variétés de l'organisation

Comme l'ont montré les historiens de la biologie (voir, en particulier, Daudin, 1926-1927; Balan, 1979), l’anatomie comparée commence à occuper une place dominante dans les sciences de la vie à partir des changements décisifs qui s'opèrent en histoire naturelle à la fin du dix-huitième siècle. Les travaux de Cuvier, en particulier, sans être bien entendu les seuls - on peut citer également ceux de Jean-Baptiste de Lamarck, Johann Friedrich Blumenbach ou d’Étienne Geoffroy Saint-Hilaire (Daudin, 1926-1927; Balan, 1979) - jouent en la matière un rôle très important.

Au risque de simplifier une histoire fort complexe, on peut dire quavant les modifications majeures introduites par cette anatomie comparée, la diversité des êtres vivants est généralement regardée par les naturalistes comme le produit d'une loi de combinaison exhaustive entre toutes les formes d'organes imaginables. Aux

3 Cette querelle doit une partie de sa célébrité au fait qu'elle a été retranscrite par Goethe. Elle joue également un rôle très important, par exemple, dans l'œuvre de Balzac. 
yeux de ces savants, il y avait autant d'êtres vivants qu'il y avait de combinaisons possibles entre toutes les formes de parties organiques susceptibles d'exister. La logique du vivant était ainsi enfermée dans cette logique combinatoire.

Progressivement sapée durant les dernières décennies du dix-huitième siècle, cette conception de la diversité de la vie est définitivement battue en brèche dans les années I790 (voir, en particulier, Balan, 1979). S’impose alors chez les naturalistes l'idée selon laquelle les combinaisons possibles entre organes ne peuvent infiniment varier, parce que le vivant est fondamentalement organisé. Cette exigence d'organisation fait que les organes sont morphologiquement et physiologiquement corrélés et subordonnés les uns aux autres. Certains agencements organiques sont impossibles, d'autres, nécessaires. Chez les mammifères, par exemple, dit Cuvier, le mode de locomotion est sous la dépendance du mode de nutrition: un animal «destiné à vivre de chair devait être muni des moyens d'attaquer et de vaincre»; celui qui doit vivre de fruits doit pouvoir grimper aux arbres; l'herbivore, au contraire, doit pouvoir rester collé à terre (I795, p. 6). Aussi, les modalités organiques sous lesquelles est accomplie la locomotion sont-elles subordonnées à celles sous lesquelles est accomplie la nutrition. Dans un tel cadre, la tâche du naturaliste se trouve donc encadrée par une nouvelle conception des espèces et du travail de classification. Il y a des espèces, une diversité du vivant, parce qu'il y a une diversité d'organisations. Classer les êtres vivants, c'est donc désormais les ranger en groupes et sous-groupes en se fondant sur la forme de l'ensemble des parties constitutives du corps, lesquelles sont, dans chaque espèce, considérées comme corrélées les unes aux autres.

Dès la fin du dix-huitième siècle, la plupart des naturalistes adhèrent globalement à ces principes classificatoires. L'histoire naturelle tend ainsi à devenir une «science de l'organisation» ou «anatomie comparée». Le sens accordé à la notion d'organisation demeure, certes, variable d'un savant à l'autre. Toutefois, une interprétation de cette notion devient peu à peu dominante à partir des années i83o. Elle s'avérera ultérieurement capitale dans le développement des sciences sociales (Guillo, 2003). On trouve cette interprétation chez des savants qui associent étroitement le thème de l'organisation et de la classification de ses variétés à celui du développement, tel qu'il a été conceptualisé 
dans l'étude des embryons, en particulier par Étienne Geoffroy Saint-Hilaire, Karl Ernst von Baer ou encore Henri Milne Edwards (voir Balan, 1979). Par-delà leurs divergences, ces naturalistes partagent l'idée selon laquelle la diversité du vivant doit être comprise comme le résultat des nécessités afférentes au développement de l'organisation vivante. Dans un tel cadre, le développement est conçu comme un processus de complexification ou de différenciation à travers lequel sont produits les corps organisés. Pour ces savants, s'il existe une variété d'espèces vivantes, s'il existe des espèces fossiles éteintes et s'il existe différents stades embryonnaires pour chaque espèce, c'est parce que les êtres vivants sont soumis à cette loi universelle, qui commande la production et le devenir de toutes les «organisations» biologiques - embryons, individus adultes, espèces, actuelles ou passées. Les étapes de ce processus sont déterminées et prescrites a priori par la série ascendante des corps organisés, couronnée par l'espèce humaine, organisation considérée comme la plus raffinée et la plus complexe. Selon ces savants, tous les embryons suivent donc ce même chemin de développement par complexification : simplement, chacun d'entre eux s'arrête plus ou moins tôt, au stade où se situe son espèce dans cette série linéaire et ascendante. Et les espèces se sont développées au fil du temps suivant un processus analogue: pour produire les êtres vivants les plus complexes, en particulier l'homme, qui surplombe par son éminence tout le règne vivant, la nature devait procéder par complexification progressive d'êtres plus simples. Tel est le sens du transformisme qui se développe au dix-neuvième siècle, avant même Darwin, par exemple chez Lamarck et Geoffroy Saint-Hilaire (voir, par exemple, Lamarck, I990; Geoffroy Saint-Hilaire, I830)4 .

Le darwinisme, comme on va le voir, dessine une conception de l'évolution radicalement différente. L'histoire de l'idée d'évolution est passablement obscurcie par le fait qu'elle est associée, tout au long du dix-neuvième siècle - y compris parfois par Darwin lui-même - à cette conception téléologique, anthropocentrique, essentialiste et scalaire de la diversité et du devenir du vivant. Ceci tient largement au conflit qui occupe le devant de la scène dans la seconde moitié du siècle: l'opposition entre le fixisme et le transformisme. Toutefois, au plan conceptuel, le transformisme n'est pas - et de très loin - l'apport le plus important et le plus original de Darwin. La révolution qu'il ouvre tient à la rupture radicale qu'il inaugure avec la conception essentialiste de l'espèce, l'échelle des êtres, l'anthropocentrisme et la téléologie, schèmes qui sont à l'inverse centraux dans les thèses transformistes de Lamarck ou de Geoffroy Saint-Hilaire: voir Balan, 1979; Gayon, 1992; Mayr, 200I. Nous reviendrons sur ce point capital dans la seconde partie. 
En résumé, dans ces théories, l'organisation est regardée non plus comme un état, mais comme un processus. La série ascendante des formes organisées, ou si l'on veut, la classification linéaire des types d'organisation, est projetée dans le temps et devient la trame d'un développement orienté vers la réalisation de formes organisées jugées supérieures, car plus complexes et raffinées. Le devenir du vivant peut alors apparaître comme une nécessité et un progrès, qui se laisse lire dans la forme des corps. Ce progrès a des indices matériels, donc susceptibles d'être considérés comme objectifs : le degré de complexité ou de différenciation du corps, qui marque un stade de développement. Le primitif devient alors l'inférieur. Sa forme et son existence n'ont de sens que par rapport à un état supérieur qu'il prophétise, dont il n’est que l'esquisse ou le vestige.

À mesure que le transformisme s'impose, tout au long de la seconde moitié du dix-neuvième siècle, deux causes possibles sont assignées à ce développement ou cette évolution du vivant, les partisans de chacune d'entre elles défendant farouchement leur point de vue. La première est «l'hérédité des caractères acquis »: selon cette hypothèse, volontiers qualifiée de «lamarckienne», l'individu peut transmettre à sa descendance les modifications qui ont affecté son organisme au cours de son existence; l'évolution est donc ici conçue comme la conséquence de l'adaptation progressive directe de chaque lignée à son environnement, à travers l’accumulation par transmission, au fil des générations, des modifications adaptives individuelles. La seconde est la «sélection naturelle», telle qu'elle est interprétée dans le sillage de l'anatomie comparée : selon cette hypothèse, qualifiée quant à elle de «darwinienne», les modifications qui affectent le corps d'un individu au cours de son existence ne se transmettent pas à sa progéniture; l'évolution est alors considérée comme la conséquence de l'extinction des lignées moins adaptées que les autres 5 .

5 Sur ce point, on pourra se reporter, en particulier, à Spencer, qui présente de telles vues de façon synthétique, en considérant qu'il y a là deux mécanismes à l'œuvre dans l'évolution. II les nomme respectivement «adaptation indirecte» et «adaptation directe» (Spencer, 1864). Sur tous ces points, voir Gayon, 1992; Bernardini, 1997; Mayr, 200I ; Guillo, 2003. 
Au total, se déploie donc à partir de cette anatomie comparée une conception transformiste du vivant, articulée autour d'une conception scalaire, hiérarchique, anthropocentrique, téléologique et essentialiste des espèces et de leur devenir.

\section{I.2. LE NATURALISME DIRECT AU DIX-NEUVIÈME SIÈCLE L'«anthropologie» issue de l'anatomie comparée}

Ces vues générales développées dans les sciences de la vie sur l'organisation, le développement et la classification sont tout d’abord étendues à l'homme à l'intérieur même de cette histoire naturelle devenue anatomie comparée. Tel est le premier usage de ces concepts issus des sciences de la vie, qui est un usage direct : les phénomènes biologiques sont ici considérés comme étant causalement impliqués dans les mécanismes qui commandent les faits sociaux.

Ainsi, dès la fin du dix-huitième siècle, nombre de naturalistes commencent à traiter l'être humain, dans leur travail taxinomique, comme une espèce parmi d’autres. Reprenant le projet, appelé de ses vœux par Buffon, d'une «histoire naturelle de l'homme» (Blanckaert, 1989), ils lui appliquent les principes de cette anatomie comparée non seulement pour lui assigner sa place dans la classification et dans le développement global du vivant - presque invariablement, tout en haut de la «série zoologique »- mais également pour décrire les différents types dont l'espèce humaine est censée se composer (Bernardini, I997; Guillo, 2003). Armés du langage de l’anatomie comparée, ces savants décrivent donc l'humanité comme un ensemble subdivisé en groupes nettement séparés les uns des autres par des «organisations», des types anatomo-physiologiques différents, qui dessinent des «races» humaines. Le critère invoqué pour justifier l'existence de ces types et les classer est, comme pour les animaux, le degré de complexité ou de différenciation prêté à l'organisation en général, et, plus spécifiquement, aux parties du corps que l'on regarde comme les substrats des facultés jugées davantage «élevées », «civilisées », en particulier le système nerveux. Car comme chez les animaux, l'organisation est considérée comme le substrat matériel de penchants et de facultés d’autant plus raffinés et subtils qu'elle est elle-même complexe et différenciée. Ce critère unique et quantifiable permet de ranger les «types humains» ainsi dégagés, tout comme on le fait 
pour les animaux, sur une ligne ascendante. Il existe ainsi, pour ces savants, une «série » hiérarchisée, une «échelle» des «races» humaines, qui constitue le tronçon terminal de la «série zoologique». Cette «série anthropologique» est invariablement couronnée par les classes supérieures de la « race » blanche occidentale, qui sont censées avoir une organisation corporelle plus complexe et moins fruste que les autres. Dans ces classifications linéaires et scalaires, les « races », les «types humains », les plus «bas » jouxtent les singes supérieurs et sont considérés comme des variétés, voire des espèces, qui s'en distinguent à peine (Tort ed, I992; Bernardini, I997). Les fous, les criminels, les prostituées ou les génies y trouveront également leur place, conformément à la particularité des traits que l'on croit déceler dans leur organisation et des dispositions que celle-ci est censée commander ${ }^{6}$.

Comme la «série zoologique» - dont elle constitue le tronçon terminal - cette «série anthropologique» est regardée comme le substrat d’un développement ascendant et progressif orienté de toute nécessité vers la réalisation des formes humaines considérées comme supérieures. Les «types» ou les «races» jugées inférieures sont donc de simples stades, des esquisses intermédiaires, dans la réalisation de ce qui constitue la finalité du développement du monde vivant: la formation de l'être humain le plus accompli, qu'incarne presque parfaitement, pour ces savants, l’homme des classes supérieures des sociétés industrielles. Celui-ci surplombe l'humanité, par un degré de développement inscrit dans l'organisation de son corps, tout comme l'homme surplombe les animaux.

Comme pour les animaux, encore, deux causes possibles sont assignées à ce développement ascendant de l'humanité, que l'on soit monogéniste ou polygéniste à propos de la généalogie des races humaines: la sélection naturelle, d'une part, les défenseurs de cette hypothèse soutenant que le progrès évolutif est assuré par la disparition des lignées humaines héréditaires les moins adaptées, cette disparition s'opérant par la concurrence entre individus ou par la guerre (les eugénistes, et des penseurs comme Georges Vacher de Lapouge, par exemple); et l'hérédité des caractères acquis, d’autre part, les 
tenants de cette hypothèse insistant sur la possibilité de faire progresser au fil des générations une même lignée d’individus par l'action d’un environnement éducatif et hygiénique sain (Émile Durkheim, Pierre Kropotkine, par exemple : voir Bernardini, I997; Guillo, 2003).

Tel est donc le vaste cadre lexical dans lequel se déploie le champ disciplinaire qui commence à s'institutionnaliser en France à partir des années i 860 sous le nom d'«anthropologie», sous l'impulsion, notamment, du médecin Paul Broca et du naturaliste Armand de Quatrefages (Blanckaert, 1989). Ce qu'il importe de souligner ici, est que cette anthropologie naît très directement de l'anatomie comparée et déploie, à travers l'extension à l'homme de ce lexique bâti en histoire naturelle, une forme de naturalisme direct. De Julien-Joseph Virey ou Bernard de Lacépède à Georges Vacher de Lapouge, en passant par Paul Broca, Cesare Lombroso ou Paul Topinard, les différences culturelles, sociales, sexuelles, ainsi que les phénomènes sociaux comme le crime, la prostitution ou le génie, sont expliqués à partir de dispositions ancrées dans une «organisation» corporelle typique, dont on s'efforce de repérer les «caractères indicateurs » à la surface des corps.

Cet usage direct des concepts de l'anatomie comparée est toutefois bien loin d'être le seul dans la sociologie et l'anthropologie naissantes. Certains auteurs, le plus souvent philosophes ou sociologues, en particulier Charles-Henri de SaintSimon et, surtout, Auguste Comte, Herbert Spencer, Alfred Espinas ou encore Émile Durkheim, s'en servent également tout au long du siècle d'une manière fort différente. Non plus en appliquant directement à l'espèce humaine le schéma développé pour les animaux, leur classification et l'interprétation de leurs dispositions et penchants; mais en utilisant les concepts de l'anatomie comparée pour déployer sous une forme rénovée les millénaires métaphores organiques de la société. Ainsi se développe un naturalisme analogique, qui doit être soigneusement distingué du naturalisme direct évoqué dans les lignes qui précèdent, même si l'un et l'autre peuvent cohabiter sans difficulté dans une même œuvre (voir, par exemple, Spencer, I876-1896, ou encore Durkheim, 1986). 


\section{I.3. LES USAGES ANALOGIQUES \\ DES CONCEPTS DE L'ANATOMIE COMPARÉE}

La sociologie comme science de l'«organisation sociale »

L’anatomie comparée constitue le réservoir lexical dans lequel les sociologues et les philosophes du dix-neuvième siècle puisent très explicitement les concepts biologiques au moyen desquels ils dressent des passerelles analogiques entre la société et le corps vivant, entre ce qu'ils nomment l'«organisation» des corps vivants et l'«organisation» des sociétés, dans le but de bâtir une «science» sociale?

On retrouve ainsi, tout d'abord, chez ces penseurs, le souci d'élaborer une classification des types d'«organisations sociales». Comme les individus, les sociétés sont donc rangées selon le critère morphologique du degré de complexité ou de différenciation internes, c'est-à-dire, pour ces sociologues et philosophes, selon le degré de division du travail (Spencer, I876-ı896; Durkheim, 1986). Ces classifications dessinent une hiérarchie des sociétés : les plus simples sont les sociétés jugées peu différenciées, «inférieures», «primitives ». Elles sont réputées se composer d'individus dont l'« organisation corporelle», en particulier psychique, est elle-même assez fruste et peu complexe. Ensuite, exactement comme la «série zoologique» et la «série anthropologique », la «série sociale » est présentée comme la trame nécessaire du devenir global des sociétés humaines. Comme toutes les «organisations», les sociétés obéissent donc à une loi de développement, qui les astreint globalement à passer au fil du temps à des formes plus différenciées, ou à disparaître. Et ce développement a deux moteurs analogues à ceux qui commandent les séries zoologique et anthropologique: la sélection naturelle entre les sociétés - la guerre et la conquête -, pour les uns, et l'adaptation directe progressive de chaque société à son environnement, à travers la transmission pacifique de génération en génération des adaptations qu'elle a acquises, pour les autres ${ }^{8}$.

7 Comte dédie son Cours au naturaliste Henri de Blainville, et reprend ses vues sur la «série zoologique». Spencer s'appuie abondamment sur les représentants de l'anatomie et de l'embryologie comparées de son temps, notamment Henri Milne Edwards et Karl Ernst von Baer. Durkheim reprend à son compte toutes ces références, par exemple, dès l'ouverture de La Division du travail social (1988). Sur tous ces points, voir Vatin, 2005; Guillo, 2003, 2006.

8 Les débats sont vifs, dans la seconde moitié du siècle, à l'intérieur de l'organicisme et du 
Ce succès du langage de l'anatomie comparée auprès des sociologues et des philosophes ne doit pas surprendre. Il n'est pas seulement le fruit de l'inventivité ou de la puissance de généralisation métaphysique de penseurs comme Comte ou Spencer. En réalité, dès le premier tiers du dix-neuvième siècle, les naturalistes eux-mêmes avaient porté les thèmes de l'organisation et du développement à un si haut degré d’abstraction en biologie, que leurs recherches suggéraient les possibilités d'une extension heuristique de leurs modèles à la société, quand elles ne développaient pas explicitement l’analogie elles-mêmes9.

En résumé, on observe au dix-neuvième siècle, d'une part, une application à l'homme très directe des concepts des sciences de la vie, qui mène à une «anthropologie», souvent franchement réductionniste et, d'autre part, un usage analogique des mêmes concepts, qui conduit à la constitution d'une «sociologie». Ce constat étant effectué, il devient possible de reconstituer la cosmologie d'ensemble, le système du monde ou, si l'on veut, le champ de pensée sous-jacent, commun à la grande majorité de ces différents discours. À l'intérieur de ce vaste champ de pensée, sécrété par le lexique de l’anatomie comparée, se déploient des modèles explicatifs qui, parfois, comme on va le voir, s'opposent franchement les uns aux autres.

\section{I.4. LA COSMOLOGIE PROPRE AUX USAGES SOCIO-ANTHROPO- LOGIQUES DES SCIENCES DE LA VIE AU DIX-NEUVIÈME SIÈCLE} La logique généralisée de l'organisation

Ce champ de pensée peut être décrit comme une logique généralisée de l'organisation (Guillo, 2003). Dans un tel cadre, le monde est conçu comme un vaste ensemble d’agrégats plus ou moins complexes et différenciés, c'est-à-dire plus ou moins organisés. Ces différents agrégats se laissent ranger en une série linéaire et ascen-

darwinisme social, à propos de la pondération qu'il convient d'accorder à chacun de ces de deux facteurs dans le développement des sociétés (sur ces points, voir Conry, 1974; Tort [ed.], 1992; Bernardini, 1997).

9 En particulier, Henri Milne Edwards, référence centrale de Spencer, mais également d'Espinas et de Durkheim sur ce thème, explique dès les années 1830 la diversité des formes vivantes en s'appuyant sur ce qu'il nomme la «division physiologique du travail (Milne Edwards, |85I). Sur ce point, voir Guillo, 2003; Vatin, 2005. Contrairement à une idée reçue, les échanges entre les sciences de la vie et les sciences sociales sont donc ici à double sens. 
dante, qui va des agrégats minéraux, jusqu'aux sociétés occidentales modernes, en passant par les êtres vivants. Tout d'abord, en se combinant les unes avec les autres, les particules chimiques s'organisent et forment les êtres vivants les plus sommaires, à peine différenciés. Ainsi s'ouvre la «série botanique», puis la «série zoologique», qui marque une progression jusqu’à l'homme, être vivant doté de l'organisation la plus complexe. Le dernier segment de cette «série zoologique» dessine donc une «série anthropologique», qui distribue la hiérarchie des «races» humaines. En se combinant les uns avec les autres, les êtres humains forment à leur tour des organisations d'un degré de complexité supérieur aux corps organiques : les sociétés. Ainsi la grande série des êtres organisés se poursuit-elle avec la série des «organisations sociales», couronnée par les sociétés industrielles (voir, par exemple, Comte, I830-I842; Spencer I876-I896; Durkheim, 1986). Au total, cette longue série étagée dessine le chemin nécessaire du développement assigné à tous les êtres qui composent le monde.

Comme on peut en juger, les notions d’organisation, de série et de développement jouent le rôle d'intégrateurs conceptuels dans l'espace du savoir qui se dessine à partir de l'extension à toute chose des concepts de l'anatomie comparée. Chaque segment de la série dessine les contours d’une discipline - zoologie, anthropologie, sociologie. En retour, ces disciplines se trouvent fortement intégrées les unes aux autres par l'unité des concepts généraux employés pour décrire leurs objets respectifs et les délimiter, et par les correspondances et les circulations incessantes de concepts que cette unité lexicale suggère, offrant ainsi de multiples prises aux esprits en quête d’analogies et de généralisations transdisciplinaires. La philosophie peut alors disserter sur le principe abstrait qui parait commander le monde : l'organisation (voir Comte, I830-1842; Spencer; I876-1896, I903).

\section{I.5. UN CHAMP D'ACTUALISATION COMMUN POUR DES THÉORIES PARFOIS ANTAGONISTES}

Il est essentiel de souligner, une fois encore, que le champ de pensée dont il est ici question est d'une extension plus vaste et d'un degré de généralité beaucoup plus élevé que ceux d’une théorie ou d’une idéologie politique définies. Il instaure un langage commun et, à travers lui, dessine des voies d'échanges 
spécifiques entre champs disciplinaires, ouvre la possibilité de généralisations abstraites. Surtout, il dessine des polarités, trace des lignes d'opposition qui rendent possible la formulation, en son sein même, de partis pris théoriques et idéologiques antagonistes. Ainsi s'opposent, par exemple, dans la seconde moitié du siècle, les penseurs convaincus que le développement des organisations individuelles tient à l'action de la sélection naturelle de souches héréditaires, et ceux qui, à l'opposé, considèrent que l'action du milieu est décisive; ou encore les sociologues individualistes, comme Spencer, et les sociologues holistes, comme Durkheim. Ces oppositions perdureront au vingtième siècle: mais elles seront alors formulées dans un autre langage, qui ne les rattachera plus au champ de pensée issu de l’anatomie comparée.

Plus largement, de vastes ensembles théoriques antagonistes se développent à partir des deux usages de l’anatomie comparée relevés dans les lignes qui précèdent: l'usage direct, d'une part, et l'usage analogique, d'autre part. Comme on l’a souligné, certaines théories prétendent expliquer les phénomènes sociaux humains en invoquant les dispositions biologiques des individus. C'est le cas, en particulier, de l'anthropologie des naturalistes ou encore de l'anthropologie criminelle italienne. Mais, d'autres, tout à l'opposé, s'appuient sur l'analogie entre le corps et la société - c'est-à-dire entre «l'organisation biologique » de l'individu et «l'organisation sociale», pour justifier, précisément, l’impossibilité d'expliquer les faits sociaux à partir de dispositions individuelles, et donc de penchants ancrés dans le corps, dans la biologie. Tel est le point de vue défendu avec force par Durkheim : «il y a entre la psychologie et la sociologie, dit-il, la même solution de continuité qu'entre la biologie et les sciences physico-chimiques. Par conséquent, toutes les fois qu’un phénomène social est directement expliqué par un phénomène psychique, on peut être assuré que l'explication est fausse » (1988, p. 197). Tout comme l'organisme vivant obéit à des lois propres, sui generis, et ne se laisse pas expliquer par les propriétés des éléments chimiques qui le composent, les phénomènes de l'organisation sociale ne se laissent pas expliquer par les lois auxquelles obéissent les éléments dont il est formé, c'est-à-dire les lois «organico-psychiques » qui commandent le corps individuel humain. 
En d'autres termes - et c'est là un point essentiel dans le cadre de la présente enquête -, la critique du naturalisme direct proposée par Durkheim s'effectue à travers la mobilisation des mêmes concepts biologiques que ceux qui ont servi à bâtir ce naturalisme, et s'inscrit par conséquent dans le même champ de pensée que lui (voir Guillo, 2006). Durkheim bâtit cette critique en se fondant sur un argumentaire qui consiste à rendre étanches les lignes de démarcation entre les différents niveaux de la logique généralisée de l'organisation. D’autres penseurs, en particulier Spencer, choisissent, quant à eux, une position intermédiaire, en rendant la frontière entre les niveaux d'organisation plus poreuse. À leurs yeux, les faits sociaux ont une autonomie épistémologique relative. Toutefois, ontologiquement, ils sont réductibles aux causes qui déterminent les conduites individuelles (voir, par exemple, Spencer, 1903).

En résumé, les discours qui proposent, au dix-neuvième siècle, de bâtir une science de l'homme et de la société en s'appuyant, d'une manière ou d'une autre, sur la biologie, dessinent une constellation de théories variées, parfois opposées, qui s'actualisent toutefois dans un champ de pensée sous-jacent commun identique, dont les concepts essentiels sont issus de l'anatomie comparée. Ces concepts sont principalement l'organisation, la classification et le développement. À travers les différentes interprétations dont ils sont l'objet, ils sécrètent donc, conjointement, un naturalisme réductionniste et un naturalisme analogique, le second allant parfois à l'encontre des explications proposées dans le sillage du premier à propos des phénomènes sociaux. Dernier élément capital, les théories sociologiques qui se déploient dans ce champ de pensée, qu'elles soient réductionnistes, ou non, ne peuvent éviter de souscrire à une conception de l'homme et de la société téléologique, essentialiste et hiérarchique, dans la mesure où le modèle biologique de référence - celui qui est issu de l'anatomie comparée - est intimement articulé autour de ces schèmes.

Comme on va tenter maintenant de le montrer, un nouveau champ de pensée articulant le biologique et le social s'est déployé à partir des sciences de la vie depuis les années 1970. Toutefois, le paradigme biologique de référence de ce nouveau naturalisme est fort différent de celui du dix-neuvième siècle, ce qui a des conséquences capitales pour les théories sociologiques et anthropologiques qui s'en inspirent. La notion générale centrale n’est plus, cette fois, l’organisation, mais le réplicateur. 


\section{LES USAGES CONTEMPORAINS DE LA BIOLOGIE EN SCIENCES SOCIALES La logique des réplicateurs \\ 2.I. LA SYNTHÈSE NÉO-DARWINIENNE}

Les théories socio-anthropologiques naturalistes contemporaines s'appuient très majoritairement sur le paradigme biologique qui domine aujourd'hui la biologie de l'évolution : la «synthèse néo-darwinienne» ou «théorie synthétique de l'évolution» (voir Mayr, 200I). Dans sa version la plus générale, ce paradigme s'appuie sur quelques propositions essentielles, qui peuvent être résumées de la façon suivante.

Tout d'abord, première proposition, les êtres vivants sont tous individuellement liés les uns aux autres au sein d’un immense arbre généalogique, qui a un point d'origine probablement unique. En d'autres termes, les êtres vivants passés et actuels sont le produit d'un processus de descendance avec modification, qui a débuté avec l'apparition d'un ancêtre commun à tous.

Second point, pour comprendre ce processus, il faut porter le regard non pas sur l'espèce, considérée comme une essence ou un type morpho-physiologique commun à différents individus, mais sur la population. Une population est un ensemble d'individus physiologiquement capables de se reproduire les uns avec les autres, et qui en ont, en outre, l'opportunité concrète. En d'autres termes, des individus d'une même espèce, au sens morpho-physiologique, séparés par une barrière géographique infranchissable, comme une montagne, n’appartiennent pas à une même population. Une population vit au sein d'une même aire écologique singulière, qui la caractérise en propre. Dans un tel cadre, l'espèce, entité abstraite, n'est donc plus le siège effectif de l'évolution. Ce qui évolue, ce sont les populations concrètes, composées d'individus porteurs de traits héréditaires susceptibles de varier légèrement d'un membre à l'autre. Tel est l'apport essentiel de Darwin (1992) : avoir introduit en biologie la «pensée populationnelle» (Mayr, 200I). 
Les populations évoluent suivant deux modalités générales. La première est la transformation graduelle interne. Ici encore, le raisonnement n'est plus centré sur l'espèce, comme essence ou type supposé commun à un certain nombre d'individus, mais sur la distribution statistique concrète des traits individuels héréditaires au sein d'une population - par exemple, la taille, la couleur ou la longueur du pelage, la forme des yeux, la formes des ailes, etc. De fait, la distribution de ces traits dans une population varie immanquablement au fil des générations. Certains traits disparaissent, d’autre apparaissent; d’autres encore sont portés par un nombre plus faible d'individus qu'auparavant, tandis que certains voient leur effectif augmenter. En ce sens, les populations se transforment au cours du temps.

Cette transformation est la conséquence de plusieurs facteurs (Sober, 1984; Mayr, 200I). Le facteur le plus volontiers évoqué, mais également le plus controversé, est la sélection naturelle. Une bonne partie des débats qui ont agité la biologie de l'évolution depuis un demi-siècle concernent le poids qu'il convient de lui accorder, certains biologistes considérant qu'elle constitue le facteur majeur de la transformation graduelle des populations (voir, par exemple, Williams, I966; Dawkins, I976; Mayr, 200I), d’autres soutenant que des facteurs aux conséquences contingentes, non nécessairement adaptatives, ont un poids au moins aussi décisif - mutations neutres, migrations et dérive génétique notamment (Gould, 1979; Kimura, 1983). Quoi qu’il en soit, la sélection naturelle est de très loin le mécanisme privilégié par les chercheurs qui proposent de s'appuyer sur la théorie de l'évolution pour expliquer les faits sociaux et culturels humains.

Dans la «synthèse néo-darwinienne», la sélection naturelle est conçue comme un processus composé de deux étapes bien distinctes. La première est la production à chaque génération de nouveautés génétiques, par mutation ou recombinaison; la seconde est la reproduction différentielle des porteurs de la variante nouvelle d'un gène ou d'une combinaison de gènes, d'une part, et des porteurs de la variante ancienne, d'autre part. Prenons l'exemple d'une population de seiches. Supposons qu’une mutation génétique survienne chez un individu sur le gène qui commande la couleur de la peau. Supposons que cette mutation donne une couleur qui se voit nettement sur le sable. Cet indi- 
vidu aura en probabilité moins de descendants que ses congénères de la même population, car il sera davantage exposé aux prédateurs. Supposons maintenant un autre gène mutant, qui donne une couleur de peau plus proche de la teinte du sable que celle des autres seiches de la population. L'individu porteur de cette mutation aura en probabilité plus de descendants que ses congénères. Ses descendants porteurs de ce gène auront à leur tour plus de rejetons, et ainsi de suite. Au fil des générations, la fréquence de cette variante du gène codant la couleur de la peau augmentera dans cette population, tandis que corrélativement la fréquence de l'autre variante diminuera, peut-être jusquà disparaître, les effectifs globaux de la population ne pouvant augmenter au-delà d’une certaine limite. Ainsi la population se sera-t-elle transformée, à travers ce processus de sélection naturelle, qui est fondamentalement un processus aveugle de reproduction différentielle entre des individus porteurs de variantes génétiques qui ne donnent pas une égale probabilité d'avoir des descendants. Tel est donc l'un des facteurs essentiels qui commandent le processus de transformation graduelle interne des populations biologiques.

Le second processus à travers lequel les populations évoluent, selon les tenants de la «synthèse néo-darwinienne», est la spéciation (Mayr, 200I). Lorsqu'une population biologique est scindée en deux ensembles distincts, sans contacts l'un avec l'autre, comme c'est le cas, par exemple, lorsqu'une barrière naturelle surgit - faille profonde, montagne, détroit rempli d'eau les deux populations ainsi formées se transforment ensuite chacune de leur côté, conformément aux mécanismes évolutifs évoqués dans le paragraphe précédent. Lorsque la séparation est suffisamment longue, la divergence entre les caractéristiques morphologiques et physiologiques des deux populations peut être telle que les individus qui les composent respectivement ne sont plus interféconds. Deux espèces différentes ont alors vu le jour. Telle est la raison pour laquelle la classification du vivant a une forme arborescente. Chaque embranchement correspond à une spéciation passée, comme ce fut le cas, par exemple, lorsque la population formée par les ancêtres que nous partageons avec les chimpanzés s'est scindée en deux groupes qui se sont ensuite transformés séparément chacun de leur côté. 
Tels sont donc, dans leurs grandes lignes, les principes essentiels de la «synthèse néo-darwinienne ». Comme on peut en juger, ce paradigme dessine une conception de la nature et du devenir du vivant radicalement différente de celle qui se déploie dans le sillage de l’anatomie comparée du dix-neuvième siècle $-y$ compris de ses variantes transformistes - sur des points capitaux. Tout d’abord, l'homme perd la place éminente qui était auparavant la sienne dans les sciences de la vie. Car l'évolution n'est plus désormais le passage à l'être progressif d'un ordre hiérarchique des espèces inscrit dans une loi de succession définie a priori - selon le degré de complexité croissante du corps - et dirigée inéluctablement vers l’avènement de l'homme, forme supérieure à toute les autres. L'évolution est désormais un processus arborescent de transformation des populations, par modification interne, divergence, redivergence, et parfois extinction, dont le déroulement obéit à tant de paramètres écologiques que l'on doit considérer qu'il suit un cours contingent. Pour cette raison, l'évolution apparaît désormais comme une véritable histoire, dans laquelle l'événement a un réel pouvoir causal (voir Balan, 1979; Gayon, 1992). Elle n’est plus un développement nécessaire, orienté vers la production d'une forme jugée supérieure. La complexité du corps n’est plus ici un gage d'éminence ontologique, car elle n'est pas un gage absolu de survie, de résistance à la sélection naturelle : certaines espèces, comme les bactéries, se perpétuent depuis la nuit des temps, alors qu'elles ont un organisme sommaire. L'homme n'est plus ici que l'extrémité d'un rameau de l'immense arbre de la vie, perdu parmi des centaines de millions d'autres. Il ne descend pas du singe, lequel serait une sorte de brouillon de l'humanité, demeuré à l'état de vestige. Il a simplement avec ce dernier des ancêtres communs. Et il peut fort bien disparaître un jour, car son existence n'est pas inscrite dans quelque loi nécessaire de l'évolution.

En somme, cette conception du vivant rompt radicalement avec l'anthropocentrisme, la conception hiérarchique et essentialiste des espèces et la téléologie, qui marquaient profondément, à l'inverse, les conceptions du vivant développées dans les sciences de la vie du dix-neuvième siècle dans le cadre de l'anatomie comparée. 


\subsection{L'EXPLICATION NÉO-DARWINIENNE DES CONDUITES SOCIALES ET DES CULTURES ANIMALES}

Ce modèle a été élaboré à l'origine pour expliquer l'évolution des caractéristiques physiques des êtres vivants. Toutefois, il a été rapidement étendu aux comportements animaux, lesquels sont intimement liés à la structure des organes qui les exécutent, et par voie de conséquence aux gènes impliqués dans leur développement et leur fonctionnement. Or jusqu’au début des années 1960, les comportements sociaux ne semblaient guère pouvoir se laisser expliquer par ce modèle. L'énigme avait déjà été relevée par Darwin luimême: comment expliquer, notamment, que les comportements altruistes au sens large, c'est-à-dire les comportements à travers lesquels un individu consacre de l'énergie à augmenter les chances de reproduction d'autrui plutôt que les siennes, ont-ils pu être retenus par la sélection naturelle? Prenons l'exemple des abeilles ou des fourmis ouvrières, qui sacrifient toute leur énergie à assurer la reproduction d'un autre individu - la reine - jusqu'à être ellesmêmes stériles. Comment ces comportements - ramener de la nourriture pour les rejetons de la reine, prendre soin des larves, défendre la ruche contre les attaques jusqu'à sacrifier sa vie, etc. - et les caractéristiques physiques qu’ils supposent ont-ils pu s'imposer dans l'évolution? L'explication par «l'utilité pour l'espèce » était certes invoquée par de nombreux éthologues, à travers le modèle de la «sélection de groupe ». Mais elle ne satisfaisait guère les plus darwiniens des biologistes. À leurs yeux, l'énigme demeurait, car quelle que soit leur utilité «pour l'espèce », de tels traits auraient dû chaque fois disparaître des populations aussitôt qu'ils étaient apparus chez un individu. En effet, les gènes qui commandent ces traits diminuent les chances de reproduction de l'individu qui les porte et augmentent celles de ses concurrents directs. Ce différentiel condamne donc immédiatement ces gènes à disparaître de la population aux générations suivantes. Comment expliquer, dès lors, le succès évolutif remporté par les comportements altruistes et, plus généralement, sociaux, que l'on observe dans nombre d'espèces ? Comment ont-ils pu passer ainsi le filtre de la sélection naturelle? 
Une première réponse à cette énigme est fournie au début des années 1960 par le biologiste William Hamilton (1964). Celui-ci fait valoir que les gènes qui codent des comportements «altruistes » peuvent se répandre dans les populations biologiques lorsque les bénéficiaires de ces comportements sont de proches parents de celui qui les possède. En effet, dans ces cas de figure, ces gènes se répandent aux générations suivantes non pas à travers les descendants directs de l'individu qui les porte - puisqu'ils diminuent la probabilité pour cet individu d'avoir des descendants - mais indirectement, en augmentant les chances de reproduction d'individus qui ont une bonne probabilité d'avoir lesdits gènes, parce qu'ils sont apparentés à l'individu «altruiste», et donc partagent une bonne partie de son génome. Ce modèle de la «sélection de parentèle» prédit donc que plus la proximité génétique entre parents proches - frères, sœurs, père, mère, cousins, etc. - est forte dans une espèce, plus la probabilité que l'on trouve des comportements sociaux dans cette espèce est importante. Or cette hypothèse a reçu une confirmation saisissante avec les hyménoptères sociaux - les fourmis, les abeilles, les guêpes, notamment. Le passage à une vie sociale avancée s'est opéré treize fois chez les insectes, dont douze fois chez les seuls hyménoptères. Or la sexualité des hyménoptères a une propriété bien spécifique: ces insectes ont une reproduction haplo-diploïdique, qui fait que les sœurs ont en moyenne $3 / 4$ de leurs gènes en commun et non simplement $1 / 2$, comme dans les espèces diploïdes, par exemple chez les mammifères.

Dans les années 1960 et 1970, d’autres modèles visant à résoudre l'énigme de l'apparition des conduites sociales dans le monde animal sont proposés, comme celui de l'«altruisme réciproque» de Robert Trivers (197I). Celui-ci propose de montrer que sous certaines conditions, de telles conduites peuvent être retenues par la sélection naturelle alors même que les individus ne sont pas apparentés.

Peu à peu se sont ainsi formés dans les années 1970 et 1980 un ensemble de modèles d'inspiration néo-darwinienne, qui ont constitué le socle de ce que l'on a nommé à partir de la publication de l'ouvrage polémique de l'entomologiste Edward Wilson la «sociobiologie animale» (1975). En somme, la «synthèse néo-darwinienne », qui dominait déjà dans le champ de l'explica- 
tion évolutive des traits morphologiques et physiologiques des êtres vivants, étend alors son empire à l'explication des comportements animaux, en particulier de leurs comportements sociaux.

\subsection{LE NATURALISME DIRECT NÉO-DARWINIEN}

Tout comme l'anatomie comparée au dix-neuvième siècle, l'explication néo-darwinienne du comportement social des animaux est alors étendue très directement à l'homme par certains sociobiologistes ${ }^{10}$, au premier rang desquels Wilson lui-même. Celui-ci propose d'expliquer les règles de l'éthique, l'évitement de l'inceste, les règles de mariage et de parenté, les liens familiaux ou encore les rôles sexuels à partir de ce paradigme (1975; 1978). Les explications consistent pour l'essentiel, chaque fois, à considérer que les normes sociales humaines sont l'expression, le plus souvent assez directe, de dispositions biologiques ancrées dans les gènes humains. Par exemple, la norme de prohibition de l'inceste ne serait que la traduction, sous forme de principe exprimé dans le langage, de l’aversion éprouvée par la majorité des humains pour l'inceste. En un mot, comme le dit le sociobiologiste David Barash (1979), les normes et, plus généralement, tout ce que l’anthropologie regroupe sous le terme de culture, se ramèneraient au «murmure intérieur chuchoté par nos gènes ». Ces gènes auraient été sélectionnés chez nos ancêtres chasseurscueilleurs du pléistocène, en vertu des avantages que procuraient les conduites qu'ils commandent dans l'environnement qui était alors le leur.

La sociobiologie humaine déclenche dès le milieu des années 1970 une vive polémique, qui se poursuit dans les années 1980. Sous le feu des critiques, elle recule peu à peu. Et sur ses décombres apparaissent de nouveaux modèles explicatifs néo-darwiniens, également assez directs, qui se développent dans le sillage des sciences cognitives, dont l'essor n’a fait que croître depuis lors. 
Les sociobiologistes ne se préoccupaient guère des mécanismes psychologiques susceptibles d'être impliqués dans les conduites humaines. Le plus souvent, ils se contentaient de montrer en quoi telle ou telle norme sociale prescrit, selon eux, des conduites adaptatives d'un point de vue néo-darwinien. Ce qui signifiait, dans leur esprit, montrer en quoi ces conduites contribuent par leurs effets, quelles que soient les intentions qui y président, à augmenter le nombre de descendants de ceux qui sont disposés à les suivre. Associées à des argumentaires visant à soutenir l'hypothèse de l'universalité de certaines normes, ces explications étaient censées démontrer que ces dispositions sont sous le contrôle de gènes sélectionnés au cours de notre passé évolutif.

Les modèles néo-darwiniens plus récents, quant à eux, centrent généralement leur explication sur les mécanismes mentaux censés commander la cognition, les émotions et les conduites humaines. Aux yeux d'une bonne partie de ces chercheurs, l'esprit est composé de modules spécialisés dans le traitement d'un type d'information - par exemple, la détection des tricheurs, la catégorisation des espèces vivantes (folkbiology), la représentation de l'esprit d’autrui (folkpsychology et theory of mind $)^{11}$. Ces modules exerceraient une contrainte sur l'architecture cognitive humaine. Ils sont considérés comme ayant, en dernière instance, un fondement génétique. Et ils doivent leur existence à leur valeur adaptative, au sens néo-darwinien. Au total, l'explication des phénomènes sociaux et culturels humains proposée par ces chercheurs s’appuie donc sur des mécanismes psychologiques dans lesquels sont impliqués des mécanismes biologiques. En ce sens, ils déploient également un naturalisme néo-darwinien direct.

Toutefois, ce courant renferme une gamme très large de théories - de la psychologie évolutionniste à l'anthropologie cognitive, en passant par l'épidémiologie des représentations de Dan Sperber (1996) -, qui ne sont pas nécessairement réductionnistes comme pouvait l'être la sociobiologie. Pour certains chercheurs, les modules peuvent en effet constituer le substrat de représentations susceptibles de varier assez fortement selon l'environnement social et

II Voir, en particulier, Barkow, Tooby et Cosmides, 1992; Medin et Atran eds, 1999; Wellman, 1990; Sperber, 1996; Pinker, 2002. 
culturel dans lequel les individus sont placés. En ce sens, les «chaînes causales de la culture» (Sperber, 1996) peuvent intégrer des représentations dont le contenu doit, au total, fort peu au gène, et beaucoup à la dynamique culturelle. Des chercheurs comme Michael Tomasello vont même plus loin, en soutenant qu'une seule disposition d'origine biologique - la capacité à se mettre à la place d'autrui - est impliquée dans le développement socio-cognitif qui permet à l'homme de déployer ses aptitudes sociales et culturelles (2004, p. 202-203).

Quoi qu'il en soit, pour les besoin de notre enquête, il suffit de noter que, par-delà leurs différences de contenu et de consistance scientifique, ces différents modèles ont en commun de proposer de rendre compte des phénomènes sociaux et culturels humains en mobilisant directement des dispositions biologiques et en inscrivant l'explication de l'émergence de ces dispositions dans une histoire évolutive néo-darwinienne. On retrouve donc bien ici, comme au dix-neuvième siècle, un naturalisme direct - plus ou moins, selon les auteurs -, bâti à partir d'un modèle devenu dominant en biologie aujourd'hui, la synthèse néo-darwinienne.

\subsection{LES USAGES ANALOGIQUES DE LA «SYNTHĖSE NÉO-DARWINIENNE» Les analogies gène/ culture}

La ressemblance avec le dix-neuvième siècle est loin de se limiter à l'émergence de ce type de naturalisme. Tout comme l'anatomie comparée, le néodarwinisme suscite dans le même mouvement un naturalisme analogique. Toutefois, dans la mesure où le modèle biologique de référence a changé, l'analogie ne porte plus sur le corps vivant, conçu comme une organisation, mais sur ce qui fait le cœur du néo-darwinisme : la logique de l'évolution des traits commandés par des gènes au sein des populations biologiques.

Ce naturalisme analogique est développé depuis les années 1970 par des chercheurs issus de disciplines variées : éthologie (Dawkins, 1976), génétique des populations (Cavalli-Sforza et Feldman, 198I ; Boyd et Richerson, 1985), philosophie (Dennett, 1996), psychologie, (Blackmore, 1999), anthropologie (Durham, I991 ; Aunger, 2002), sociologie (Runciman, 1998, 2005). Par-delà 
les différences qui les séparent, ces chercheurs considèrent tous que le naturalisme direct ne peut permettre de rendre compte des faits sociaux et culturels humains, même s'il peut valoir pour les comportements animaux. Pour défendre ce parti pris, ils reprennent à leur compte une thématique assez ancienne en la formalisant dans le langage du néo-darwinisme: la thématique d'une analogie entre l'évolution des traits biologiques et la diffusion des idées dans les groupes humains.

L’exemple le plus célèbre de ce naturalisme analogique est assurément la théorie des mèmes, ou mémétique, proposée en 1976 par l'éthologue Richard Dawkins (1976, chapitre XI). À ses yeux, l’idée réellement révolutionnaire du néo-darwinisme consiste à concevoir l'évolution comme un processus de concurrence entre les gènes, considérés comme des entités qui ont la propriété de produire des copies d'elles-mêmes, autrement dit de se répliquer et, parfois, de muter, les nouvelles variantes entrant alors en concurrence pour la réplication avec les anciennes. Or, fait valoir Dawkins, l'apparition de l'espèce humaine a créé les conditions écologiques favorables à l'émergence de «nouveaux réplicateurs », différents des gènes, mais obéissant aux mêmes mécanismes évolutifs généraux qu’eux.

En effet, dit Dawkins, les humains ont la capacité d'imiter leurs congénères. Les idées ou les pratiques ont donc, tout comme les gènes, la capacité de produire des répliques d'elles-mêmes dans le cerveau d’autrui, pour autant qu'elles s'avèrent psychologiquement attractives. Ainsi l'imitation joueraitelle dans le domaine culturel le même rôle que la réplication et la reproduction dans le domaine biologique. Comme pour le gène, poursuit Dawkins, il arrive que le processus de réplication d'une idée soit imparfait : un moine du Moyen Âge transforme une phrase d'un manuscrit qu'il recopie, un individu invente une nouvelle onomatopée, un cuisiner propose une nouvelle recette, un individu prêche qu'il n'existe qu'un seul dieu, etc... Ces mutations entrent alors en concurrence pour la réplication avec les anciennes variantes. Dans certains cas, la nouvelle variante parvient à produire plus de répliques d'ellemême que les variantes classiques, avec lesquelles elle est en concurrence : de plus en plus de cuisiniers font la nouvelle recette, plutôt que les anciennes, la 
nouvelle onomatopée est adoptée par un nombre croissant d'individus dans un groupe social, le monothéisme se développe, etc.

Au total, ces idées, ces entités culturelles - que Dawkins choisit de nommer «mèmes »- sont donc plongées, comme les gènes, dans un processus d'évolution, dans lequel la sélection par réplication différentielle joue un rôle capital. Et comme pour les gènes, ce processus commande leur contenu et l'évolution de leurs effectifs. Au total, les mécanismes évolutifs généraux qui commandent les «réplicateurs biologiques» sont donc globalement identiques à ceux qui commandent «les réplicateurs culturels $\gg^{12}$.

Telles sont les grandes lignes du naturalisme analogique actuel. Comme celui du dix-neuvième siècle - et c'est là un point essentiel -, il conduit à des modèles qui s'opposent fermement au naturalisme direct bâti à partir des concepts biologiques dont il s'inspire par analogie. En effet, aux yeux de ces chercheurs, les idées passent par imitation d'un cerveau à un autre. Ce qui implique qu'elles ne sont pas présentes dans les esprits avant ces transmissions, sous forme de dispositions cognitives innées (voir Aunger ed, 2000).

\subsection{LA COSMOLOGIE PROPRE AUX USAGES ACTUELS DE LA BIOLOGIE - LA LOGIQUE GÉNÉRALISÉE DU RÉPLICATEUR Comparaison avec le dix-neuvième siècle}

$\mathrm{Au}$ total, les deux grandes extensions socio-anthropologiques actuelles de la biologie - principalement, le naturalisme direct et le naturalisme analogique néo-darwiniens - dessinent, comme au dix-neuvième siècle, un vaste champ de pensée, engendré par l'application en cascade d'un principe qui a initialement acquis ses titres de noblesse épistémologique en biologie. Mais, la notion clé autour de laquelle s'ordonne ce principe a changé. Il ne s’agit plus de l'«organisation », mais du «réplicateur ».

12 Sur les mécanismes communs à l'évolution biologique et à l'évolution culturelle et, également, sur leurs différences - notamment le poids évolutif des mécanismes de transmission dans l'évolution culturelle, mécanismes qui sont sans effets dans le domaine biologique - voir, notamment, Cavalli Sforza et Feldman, 1981 ; Boyd et Richerson, 1985 ; Durham, 1991. 
Le monde phénoménal apparaît, ici encore, comme un ensemble dont la structure est étagée. Le premier niveau s'est dessiné au cœur des phénomènes physico-chimiques, lorsque certaines molécules ont acquis la propriété de se répliquer spontanément. Cette propriété les a plongées dans un processus d'évolution par sélection, qui a engendré un second niveau phénoménal : celui des phénomènes biologiques. Le réplicateur qui commande fondamentalement ce niveau est le gène. L'évolution des gènes a ensuite conduit certaines espèces à développer des capacités physiologiques complexes : sensations, perceptions, émotions, désirs, croyances, en d'autres termes ce que l'on appelle les phénomènes de l'esprit. Ainsi s'est formé un nouveau niveau: celui des phénomènes psychologiques. Au cœur de ce niveau, de nouveaux réplicateurs ont alors émergé - les mèmes ou réplicateurs culturels ${ }^{13}$-, dont l'existence a été rendue possible par une propriété psychologique: la faculté d'imitation, que possèdent certaines espèces, au premier rang desquelles l'être humain. Les mèmes ont évolué à leur tour, suivant des mécanismes formellement identiques, à quelques différences près, à ceux qui commandent les gènes - sélection, migration, mutations neutre, dérive, notamment (Cavalli-Sforza et Feldman, I98I ; Durham, 199I). Ainsi sont apparus les phénomènes culturels, c'est-à-dire les idées et les pratiques qui se diffusent dans les populations par transmission sociale. Cette évolution culturelle a, certes, été rendue possible par l'évolution génétique et elle lui est analogue. Toutefois, précisément parce qu'elle est commandée par un nouveau type de réplicateur, elle est autonome vis-à-vis des gènes et de leur évolution. En ce sens, la mémétique s'oppose à la sociobiologie.

Comme celui du dix-neuvième siècle, le champ de pensée qui se déploie aujourd'hui à partir de la biologie est beaucoup plus vaste et indéfini qu'une théorie. Il dessine des polarités, ouvre des débats et des points de vue possibles très variés, parfois antagonistes. L'exhumer, comme nous avons tenté de le faire, permet de comprendre la logique sous-jacente qui unit, par-delà ce qui les oppose, les différentes théories socio-anthropologiques qui invoquent

13 Les auteurs évoqués ici n'utilisent pas tous le mot «mème». Toutefois, ils adhèrent globalement au principe d'un réplicateur, au sens où l'entend le néo-darwinisme (voir par exemple Cavalli-Sforza et Feldman, 1981). 
aujourd'hui le néo-darwinisme, en montrant comment chacune d'entre elles correspond à un positionnement défini dans ce champ.

La sociobiologie humaine, tout d’abord, représente un parti pris théorique extrême, fortement réductionniste, dans lequel la spécificité des niveaux psychologiques et culturels est presque annulée. Aucune autonomie, ou presque, n'est reconnue à ces derniers : pour les sociobiologistes, il n'existe pas véritablement de réplicateur culturel.

Les modèles développés dans le sillage des sciences cognitives, quant à eux, - psychologie évolutionniste, épidémiologie des représentations, anthropologie cognitive - sont centrés sur le niveau psychologique, auquel ils accordent une épaisseur et une consistance maximales. Toutefois, les chercheurs qui s'inscrivent dans ces courants considèrent que les mécanismes mentaux qu'ils mettent au jour sont malgré tout ancrés en dernière instance dans des gènes plus ou moins fermement et directement selon les cas. Certes, ils reconnaissent que les réplicateurs culturels peuvent exister. Mais à leurs yeux, ces cas sont rares, les phénomènes culturels s'expliquant le plus souvent par des opérations mentales bien définies, et non par une imitation d'autrui (voir, par exemple, Sperber, 1996; Aunger ed, 2000). Au total, pour ces chercheurs, un substrat biologique est impliqué dans les mécanismes qui commandent les phénomènes sociaux et culturels : mais son rôle est toutefois moins direct et davantage réfracté que dans les modèles proposés par la sociobiologie.

De son côté, le naturalisme analogique accorde la primauté au niveau de réalité où se déploient les phénomènes culturels, lesquels sont considérés comme autonomes vis-à-vis des phénomènes psychologiques et biologiques. On retrouve ici une position épistémologique générale semblable à celle de Durkheim, qui mobilisait le modèle biologique dominant de son temps pour le retourner, au moyen de l'analogie, contre les partis pris réductionnistes que ce dernier inspirait directement, afin d'appuyer l'idée d'une autonomie du social vis-à-vis du psychologique et du biologique. 
Enfin, depuis le début des années 1990, certains théoriciens proposent de regarder ces différents partis pris comme autant de cas de figures susceptibles d'être rencontrés dans la réalité. Les théories évoquées dans les lignes qui précèdent sont alors considérées comme une batterie de schémas explicatifs, l'objectif étant pour le chercheur de déterminer lequel est pertinent dans chaque cas empirique concret. Tels sont les principes des théories synthétiques de la «coévolution gène / culture», comme celle qua proposée l’anthropologue William Durham (199I).

Comme on peut en juger, ce processus d'extension du néo-darwinisme à l'explication des phénomènes de l'esprit, de la société et de la culture présente de nombreuses similitudes avec la formation du vaste champ de pensée qui se dessine au dix-neuvième siècle à partir de l'anatomie comparée.

Tout d’abord, le néo-darwinisme est devenu dominant en biologie dans les disciplines chargées de rendre raison de la diversité et du devenir des espèces vivantes, comme l'anatomie comparée en son temps. Tout comme cette dernière, il a été ensuite utilisé très directement pour expliquer les phénomènes sociaux et culturels humains, ramenant ceux-ci à des dispositions d'origine biologique. Portée à un très haut degré d’abstraction, la notion sur laquelle il s'articule a ensuite sécrété un naturalisme analogique, qui s'oppose au naturalisme direct, comme l'attestent les vifs débats, depuis une dizaine d’années, entre les représentants de la psychologie évolutionniste et de l'anthropologie cognitive, d'une part, et ceux qui considèrent que l'évolution culturelle est analogue à l'évolution biologique, d'autre part ${ }^{14}$.

En outre, comme la logique généralisée de l'organisation au dix-neuvième siècle, le champ de pensée ouvert par le néo-darwinisme dessine une conception étagée des niveaux phénoménaux, homogénéisée par un langage et des concepts communs. Ce langage et ces concepts sont eux-mêmes articulés autour d'une notion d'un haut degré de généralité, qui permet une circulation ininterrompue de concepts d'un niveau à l'autre. Peints sous les couleurs d'un même lexique, les faits organiques et les faits sociaux ne peuvent alors manquer de présenter des analogies qui frappent les esprits. Certains savants -Étienne Geoffroy Saint Hilaire 
au dix-neuvième siècle, Richard Dawkins depuis une trentaine d'années - ou philosophes - Herbert Spencer hier, Daniel Dennett aujourd'hui - entreprennent de démontrer que les concepts centraux de ces théories issues initialement de la biologie constituent des principes d'une portée cosmologique très générale, qui commande l'ensemble du monde phénoménal: loi d'évolution par complexification de l'organisation, pour Spencer (1876-1896), schéma réplication / mutation / sélection, comme algorithme universel, pour Dennett (1996).

\subsection{LE NATURALISME ACTUEL PEUT ÉCHAPPER AU RÉDUCTIONNISME ET AU PRINCIPE DE HIÉRARCHIE}

De cette comparaison entre le dix-neuvième siècle et la période actuelle se dégagent deux enseignements fondamentaux, qui vont à l'encontre de thèses fréquemment défendues en sciences sociales à propos du contenu du naturalisme socio-anthropologique et de son histoire depuis deux siècles. Tout d'abord, une bonne partie des études consacrées à ces théories réduisent généralement ces dernières au naturalisme direct, considérant que les doctrines anti-réductionnistes d'une part ne peuvent être produites qu'à partir de concepts ayant une tout autre origine que les sciences de la vie, d’autre part s'opposent nécessairement à tout usage des concepts de la biologie en sciences sociales ${ }^{15}$. Or, comme on l'a vu, les modèles socio-anthropologiques inspirés par les sciences de la vie depuis deux siècles sont bien loin de se limiter à des thèses réductionnistes. Beaucoup plus profondément et subtilement, les champs de pensée issus de la biologie suscitent en leur propre sein des modèles qui vont à l'encontre du réductionnisme, à travers un naturalisme de type analogique.

Ensuite, et c'est sans doute là l'élément qu'il convient de souligner avec force, les modèles biologiques autour desquels sont respectivement bâtis les deux champs de pensée évoqués dans les lignes qui précèdent présentent, par-delà leurs ressemblances, des différences fort importantes, qui ont des conséquences

I5 C'est le cas, par exemple, d'une interprétation répandue de l'œuvre de Durkheim, interprétation dans laquelle sa défense de l'autonomie du social vis-à-vis du psychologique et du biologique est présentée comme une rupture radicale avec les schèmes de pensée issus des sciences de la vie de son temps. Cette interprétation est défendue, par exemple, par Muchielli (1998) ou encore Blanckaert (2004). Pour une critique de cette interprétation, voir Guillo, 2006. 
capitales pour les théories socio-anthropologiques bâties à partir d'eux. Comme on l’a souligné plus haut, le néo-darwinisme déploie une conception de la diversité du vivant dans laquelle les espèces ne sont plus conçues comme des types clos sur eux-mêmes, tirant leur essence et leur existence d'un ordre hiérarchique a priori inscrit dans les corps et couronné par l'homme. En d’autres termes, la diversité du vivant, et par conséquent son devenir, peuvent être désormais pensés indépendamment des schèmes essentialistes, hiérarchiques, anthropocentriques et téléologiques. Ceci implique que l'on peut aujourd'hui fort bien développer des thèses naturalistes en sciences humaines sans pour autant bâtir des modèles hantés par ces schèmes. Bien entendu, cela ne signifie pas que les discours qui invoquent aujourd'hui Darwin ou la biologie à propos de la société et de la culture humaines soient exempts de tels schèmes : de fait, nombre d'entre eux déploient implicitement, et parfois explicitement, des vues à forte charge ethnocentrique, sexiste, discriminatoire et historiciste, comme ce fut le cas avec une partie de la sociobiologie humaine. Simplement, ce n'est plus aujourd'hui une fatalité inscrite dans la théorie biologique sur laquelle s'appuie le naturalisme.

Tel n'était pas le cas pour la sociologie et l'anthropologie naturalistes du dix-neuvième siècle. Conceptualisant le vivant au moyen de la notion d'organisation, telle qu'elle apparaît dans l’anatomie comparée, le naturalisme direct ne pouvait manquer de concevoir les dispositions biologiques comme des penchants corrélés dans chaque individu à d’autres penchants bien définis, le tout formant un agencement morphologique et physiologique propre à un certain type, lisible dans l'organisation corporelle, c'est-à-dire dans la forme des parties du corps. Dans un tel cadre, l'intensité d'une disposition, par exemple, au crime, au suicide, au vol ou à la prostitution, chez un individu, le ramenait nécessairement à une organisation corporelle globale, et donc à une essence, un type particulier, qui lui assignait une place bien précise dans un ordre hiérarchique - l'échelle des êtres graduée selon le degré de complexité de l'organisation. Cette place devait, en outre, être lue comme un stade plus ou moins avancé dans un développement, orienté vers l’apparition de types humains jugés plus développés, supérieurs. Elle ne pouvait donc manquer d’apparaître comme un rang dans une échelle des dignités. Il en va de même pour le natu- 
ralisme analogique du dix-neuvième siècle, dans la mesure où il applique les mêmes schèmes aux sociétés, conçues comme des «organisations sociales». Il est voué à classer celles-ci selon un ordre hiérarchique, censé marquer des étapes dans un développement tourné de toute nécessité vers des sociétés dans lesquelles le travail est toujours plus divisé. Pour toutes ces raisons, les différents avatars du naturalisme du dix-neuvième siècle déploient nécessairement des vues à forte charge discriminatoire, raciste et sexiste.

Le naturalisme direct actuel, quant à lui, peut fort bien décrire une disposition sans considérer qu'elle est nécessairement corrélée à d’autres dispositions définies chez les individus. Et la force d'une disposition ne se laisse pas nécessairement lire avec netteté dans le corps. Par ailleurs, la présence d'une disposition ne place en rien l'individu qui la possède dans quelque échelle hiérarchique; tout simplement parce que la hiérarchie n'est plus un principe constitutif de la classification des êtres vivants. À cet égard, il est essentiel de rappeler que, dans la pensée populationnelle et la classification qui en dérive, le principe de sélection naturelle n’est l'opérateur d’aucun progrès, au sens propre du terme (Gould, I997; Mayr, 200I). Le succès d'une forme vivante sur une autre ne renvoie jamais à quelque supériorité absolue, indépendante des conditions écologiques singulières dans lesquelles vit une population: il est toujours relatif à ces conditions variables dans l'espace et dans le temps. Aucune disposition ou organisation particulière, y compris la complexité de l'esprit ou du corps, ne garantit un succès dans la concurrence pour la survie et la reproduction. D’autant plus que bien d’autres mécanismes aux résultats contingents y jouent un rôle crucial, comme les migrations, les mutations neutres ou la dérive génétique (Sober, 1984; Mayr, 200I).

On peut formuler les mêmes remarques à propos du naturalisme analogique d'aujourd'hui. Ces modèles se ramènent, en définitive, aux doctrines, classiques en sciences sociales depuis Gabriel Tarde, qui tentent de décrire la dynamique culturelle comme un processus de diffusion des idées par imitation. L'objectif est ici de rendre compte des mécanismes par lesquels telle ou telle idée ou pratique se diffuse dans un groupe social, sans établir quelque échelle de dignité entre elles. 
Ces remarques ont d'importantes conséquences pour le regard que les sciences sociales portent actuellement sur le naturalisme socio-anthropologique. Tout d’abord, elles démontrent la fragilité d'un raisonnement critique assez répandu. Ce raisonnement consiste à focaliser l'attention sur certaines affinités discursives entre les théories socio-anthropologiques naturalistes du dix-neuvième siècle et celles d'aujourd'hui, pour conclure dans un second temps que l'on doit retrouver dans les secondes les dérives idéologiques des premières, même lorsque ces dérives ne sont pas explicitement formulées. Or, comme on a tenté de le montrer, le naturalisme d'aujourd'hui s'appuie sur un modèle biologique fort différent de celui du dix-neuvième siècle; un modèle biologique, précisément, exempt des schèmes qui conduisaient nécessairement le naturalisme du dix-neuvième siècle à développer des discours hiérarchiques et historicistes sur l'être humain. Bien entendu, on retrouve de tels discours, aujourd'hui, dans certains écrits naturalistes. Mais - et c'est là un point capital - le naturalisme n'est plus aujourd'hui condamné à souscrire à de telles thèses.

En se détournant de l'analyse précise du contenu théorique des doctrines, pour se concentrer sur leurs proximités idéologiques supposées à un siècle de distance, ce type de critique du naturalisme conduit à des conclusions parfois contestables en matière d'histoire des idées et d'épistémologie. Surtout, il peut conduire à esquiver un débat proprement théorique et empirique aujourd'hui essentiel - celui que les sciences sociales doivent avoir avec les sciences de la vie -, risquant ainsi d'assurer une forme d'immunité aux versions les plus sommaires et idéologiques du naturalisme actuel.

\section{BIBLIOGRAPHIE}

AUNGER R. (ed.), 2000, Darwinizing Culture: The Status of Memetics as a Science, Oxford, Oxford University Press.

-, 2002, The Electric Meme. A New Theory of How we Think, New York, The Free Press.

BALAN B., 1979, L'Ordre et le Temps. L'anatomie comparée et l'histoire des vivants au $X I X X^{e}$ siècle, Paris, Vrin. 
BARASH D.P., 1979, The Whisperings Within: evolution and the origin of human nature, Londres, Harper \& Row.

BARKOW J.H., COSMIDES L. et TOOBY J., 1992, The Adapted Mind: evolutionary psychology and the generation of culture, Oxford, Oxford University Press.

BERNARDINI J. M., 1997, Le Darwinisme social en France. Fascination et rejet d'une idéologie (1859-1918), Paris, Éditions du CNRS.

BERTHOUD G. (dir.), 1985, Les Sciences sociales face au défi de la sociobiologie, Revue européenne des sciences sociales, XXIII-69.

BLACKMORE S., 1999, The Meme Machine, Oxford, Oxford University Press.

BLANCKAERT C., 1989, «L'Anthropologie en France, le mot et l'histoire», Bulletins et mémoires de la Société d'anthropologie de Paris, n. s., t. I, 3-4, p. 13-44. -, 2004, La Nature de la société. Organicisme et sciences sociales au XIX siècle, Paris, L'Harmattan, coll. «Histoire des sciences humaines».

BOYD R. et RICHERSON P.J., 1985, Culture and the Evolutionary Process, Chicago, The University of Chicago press.

CAVALLI-SFORZA L.L. et FELDMAN M.W., 198I, Cultural Transmission and Evolution: a Quantitative Approach, Princeton, Princeton University Press.

COMTE A., 1998 (1830-1842), Cours de philosophie positive, Paris, Hermann, 2 t.

CONRY Y., 1974, L'Introduction du darwinisme en France au XIX siècle, Paris, Vrin.

CUVIER G., 1795, Mémoire sur une nouvelle division des mammifères, extr. du Magasin encyclopédique ou Journal des Sciences, des Lettres et des Arts, $n^{\circ} \mathrm{VII}$, t.II, p. 164-190.

DAUDIN H., 1926-1927, Cuvier et Lamarck. Les classes zoologiques et l'idée de série animale (1790-1830), Paris, Félix Alcan, 2 t.

DARWIN Ch., 1992 (I859), De l'origine des espèces, Paris, Garnier-Flammarion.

DAWKINS R., 1976, The Selfish Gene, Oxford, Oxford University Press.

DENNETT D., 1996, Darwin's Dangerous Idea. Evolution and the Meaning of Life, London, Penguin Books. 
DURHAM W. H., 199I, Coevolution. Genes, Culture, and Human Diversity, Stanford, Stanford University Press.

DURKHEIM É., 1986 (I893), De la division du travail social, Paris: PUF, coll. «Quadrige». -, 1988 (1894), Les Règles de la méthode sociologique, Paris, Flammarion.

GAYON J., 1992, Darwin et l'après-Darwin: une histoire de l'hypothèse de sélection naturelle, Paris, Kimé.

GEOFFROY SAINT-HILAIRE É., 1830, Principes de philosophie zoologique, discutés en mars 1830 au sein de l'Académie royale des sciences, Paris, Pichon et Didier.

GOULD S. J., 1997 (1996), L'Éventail du vivant: le mythe du progrès, Paris, Seuil.

- et LEWONTIN R., 1979, «The Sprandels of San Marco and the Panglossian Paradigm: A Critique of the Adaptationist Programme», Proceedings of the Royal Society, B025, p. 58I-598.

GUILLO D., 2003, Les Figures de l'organisation. Sciences de la vie et sciences sociales au XIXe siècle, Paris, PUF.

-, 2006, «La Place de la biologie dans les premiers textes de Durkheim: un paradigme oublié?», Revue française de sociologie, 47-3, p.507-536.

HAMILTON W., 1964, «The genetical evolution of social behaviour. I», Journal of theoretical biology 7 -I, p. I-16.

KIMURA M., 1983, The Neutral Theory of Molecular Evolution, Cambridge, Cambridge University Press.

LAMARCK J. B. de, 1990 (I820), Système analytique des connaissances positives de I'homme, Paris, PUF.

MAYR E., 200I, What Evolution Is, New York, Basic Books.

MEDIN D. et ATRAN S. (eds), 1999, Folkbiology, Cambridge, MIT Press.

MILNE E. H., I85I, Introduction à la zoologie générale ou considérations sur les tendances de la nature dans la constitution du règne animal, Paris, Victor Masson.

MUCCHIELLI L. (dir), 1994, Histoire de la criminologie française, Paris, L'Harmattan. -, 1998, La Découverte du social. Naissance de la sociologie en France (I870-1914), Paris, La Découverte. 
PINKER S., 2002, The Blank Slate. The Modern Denial of Human Nature, Londres, Allen Lane, Penguin Books.

RUNCIMAN W.G., 1998, «The selectionist paradigm and its implications for sociology», Sociology, 32, p. 163-188.

-, 2005, «Culture does Evolve», History and Theory, 44-I, p. I-13.

SAHLINS M., 1980 (1976), Critiques de la sociobiologie. Aspects anthropologiques, Paris, Gallimard, «Bibliothèque des sciences humaines».

SOBER E., 1984, The nature of selection. Evolutionary theory in philosophical focus, Chicago, The University of Chicago Press.

SPENCER H., 1864, The principles of biology, London, William and Norgate, t. 2.

-, 1903 (1873), Introduction à la science sociale, Paris, Félix Alcan.

-, 1876-1896, The principles of sociology, London, William and Norgate.

SPERBER D., 1996, La Contagion des idées, Paris, Odile Jacob.

TOMASELLO M., 2004 (1999), Aux origines de la cognition humaine, Paris, Retz.

TORT P. (dir.), 1992, Darwinisme et société, Paris, PUF.

TRIVERS R. L., 197I, «The Evolution of Reciprocal Altruism», The Quarterly Review of Biology, $46-$ I, p. 35-57.

VATIN F., 2005, Trois essais sur la genèse de la pensée sociologique: politique, épistémologie et cosmologie, Paris, La Découverte.

WELLMAN H.M., 1990, The Child's Theory of Mind, Cambridge, MIT Press.

WILLIAMS G.C., 1966, Adaptation and natural selection, Princeton, Princeton University Press.

WILSON E. O., 1975, Sociobiology. The new synthesis, Harvard, Harvard University Press. 